\title{
Bioinspired Hydrogels for 3D Organoid Culture
}

\author{
Delphine Blondela and Matthias P. Lutolf ${ }^{\star a b}$
}

\begin{abstract}
Organoids have stepped into the limelight as unique in vitro systems for modeling organ development, function and disease. This review provides a perspective on how chemically defined, bio-inspired hydrogels could be used for replacing ill-defined matrices derived from the native extracellular matrix (ECM) that are used for generating organoids in 3D stem cell culture. In particular, we propose the use of self-healing and light-responsive matrices that should afford control over the inherently stochastic self-organization process that currently underlies organoid morphogenesis. Such designer ECMs could accelerate the translation of organoid technology from the laboratory into various real-life applications.
\end{abstract}

Keywords: ECM · Hydrogel $\cdot$ Matrigel $^{\mathrm{TM}} \cdot$ Organoid $\cdot$ Photo-patterning $\cdot$ Synthetic gel $\cdot$ Self-healing

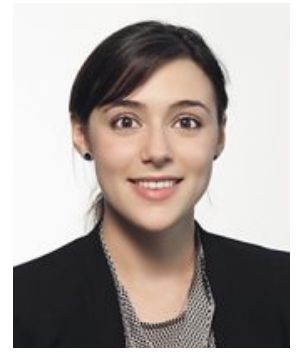

Delphine Blondel studied chemistry and chemical engineering at the École Polytechnique Fédérale de Lausanne (EPFL). She conducted her master thesis in the laboratory of Prof. David Schaffer (UC Berkeley) on the use of optogenetic methods to control signaling pathways in neural stem cells. She obtained her master degree in 2014 at EPFL. In 2015, she started her $\mathrm{PhD}$ studies under the guidance of Prof. Lutolf. Her current research focuses on the development of synthetic hydrogels as alternatives to native ECMs for organoid culture.

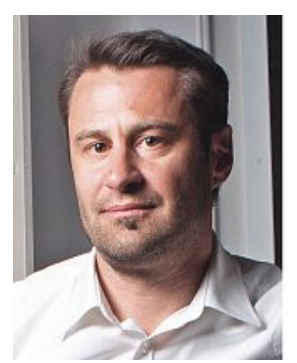

Prof. Matthias Lutolf directs the Laboratory of Stem Cell Bioengineering (LSCB) at EPFL. His highly cross-disciplinary and cutting-edge research program is focused on the development of bio- and tissue-engineering strategies for better understanding stem cell self-organization towards improved organoid culture and applicability.

\section{Introduction}

Over the past decade, tremendous progress has been made in growing in vitro relatively complex artificial tissues termed organoids. ${ }^{[1,2]}$ Organoids emerge from initially homogeneous stem cell populations that 'self-organize' into intricately structured multicellular entities that recapitulate, in 3D and at a sub-millimeter scale, key architectural and functional features of native tissues and organs. The first example of organoids generated from adult stem cells was reported by Clevers and colleagues who succeeded in coaxing mouse intestinal stem cells (ISC) into remarkably elaborate epithelial tissues. ${ }^{[3,4]}$ These 'mini-guts' are composed of so-called crypts, harboring self-renewing ISCs and Paneth (niche) cells, as well as villus-like domains, harboring the key differentiated cell types found in the intestinal epithelium. This ground- breaking work, together with landmark studies on pluripotent stem cell-derived (embryonic) organoids by the lab of Yoshiki Sasai, ${ }^{[5,6]}$ led to an explosion of activities by laboratories around the world developing protocols for organoids from multiple stem cell types. ${ }^{[7]}$ To date, numerous mouse and human organs including the intestine, stomach, colon, liver, pancreas, kidney, brain and retina, have found in vitro organoid equivalents. ${ }^{[8]}$ Besides being extremely useful as model systems for mechanistic studies on organ biology and disease, organoids promise to bridge the gap between conventional in vitro cell culture and the more complex, expensive and ethically challenged animal experimentation.

However, despite their exciting potential for new areas of applications in biomedicine, existing organoid cultures have significant shortcomings. ${ }^{[7]}$ First, the derivation of organoids typically requires animal-derived ECMs such as Matrigel $^{\mathrm{TM}}[9]$ or similar products sold under different names (e.g. Cultrex $\left.{ }^{\circledR}\right)$. The poorly defined composition and batch-to-batch variability of these biomaterials poses a problem in both basic and translational research, as we discuss below. Second, and at least in part owing to these poorly defined first-generation culture systems, most organoids develop through unpredictable processes, giving rise to tissues with variable size, shape and cell-type composition. ${ }^{[10]}$ In the last few years, increasing efforts have been dedicated to develop bioinspired materials that could replace these native ECM-derived matrices, ${ }^{[11]}$ potentially generating organoid culture protocols that are more reproducible and clinically compatible. Here, we briefly discuss 3D culture matrices that are being used to generate organoids and then highlight synthetic hydrogels with the potential for improving organoid cultures.

\section{State-Of-The-Art Organoid Culture Using Naturally Derived ECMs}

Due to its tissue-like physical characteristics as well as outstanding stem cell niche signaling properties, Matrigel ${ }^{\mathrm{TM}}$ is the gold standard for organoid culture. ${ }^{[7]}$ Matrigel $^{\mathrm{TM}}$ is composed of a plethora of different basement membrane components including laminin-1, collagen IV, entactin, and heparin sulfate proteoglycans, as well as numerous growth factors. It can be conveniently 
used as a 3D cell culture scaffold by inducing crosslinking through heating of a cell-containing precursor solution from $4{ }^{\circ} \mathrm{C}$ to $37^{\circ} \mathrm{C}$. Although the advantages and applications of Matrigel ${ }^{\mathrm{TM}}$ (and related basement membrane-type gels) to culture stem cell-derived organoids are manifold, several drawbacks remain:

- The nearly unquantifiable lot-to-lot variability of Matrigel ${ }^{\mathrm{TM}}$ undermines reproducibility and thus the applicability for scaling-up organoid assays.

- Because of its complex composition, Matrigel ${ }^{\mathrm{TM}}$ is unsuitable for understanding cell signaling and unraveling the role of individual ECM components on organoid formation. Indeed, the physicochemical and cell-signaling properties of Matrigel ${ }^{\mathrm{TM}}$ cannot be easily tailored to customize it for specific stem cell and organoid types.

- Because of their temperature sensitivity and susceptibility to protease-mediated degradation, natural ECM-derived matrices such as Matrigel ${ }^{\mathrm{TM}}$ are often difficult to handle and process, precluding their use for long-term cell culture.

- When used in vivo, the animal origin of Matrigel ${ }^{\mathrm{TM}}$ may induce unwanted immunogenic responses.

Collagen type I and fibrin hydrogels are some of the Matrigel $^{\mathrm{TM}}$ alternatives that have been explored for the culture of mouse and human organoids. For example, Kuo and colleagues developed an air-liquid interface system based on collagen gels for the longterm culture of intestinal epithelia. ${ }^{[12]}$ Clevers and co-workers showed that ISCs embedded in a collagen type I gel can selforganize and form tube-shaped tissues. ${ }^{[13]}$ Recently, Broguière and colleagues demonstrated that fibrin matrices supplemented with laminin provide an excellent support for ISC proliferation and organoid formation, from both mouse and human stem cells. However, the stereotypical patterning of ISCs seen in Matrigel $^{\mathrm{TM}}$ could not yet be fully reproduced in these modified fibrin gels.[14]

\section{Organoid Derivation via Cell-Instructive Synthetic Hydrogels}

The aforementioned challenges inherent to native ECMs for organoid culture have fueled increasing efforts to design defined synthetic alternatives, in particular those based on covalently crosslinked hydrophilic polymers such as poly(ethylene glycol) (PEG). In general, such matrices have well-defined molecular architectures, resulting in excellent relationships between composition and physicochemical properties (e.g. swelling, viscoelastic-
Fig. 1. Synthetic PEG-based hydrogels engineered as niches for mouse ISC expansion and organoid formation. a) Schematic illustrating the chemical structure of the synthetic hydrogels generated by enzymatic crosslinking via the transglutaminase FXIIla. b) Summary of the matrix 'rules' regulating different steps in organoid formation. c) Intestinal organoids formed in synthetic hydrogels containing key differentiated cell types of the native tissue. a.

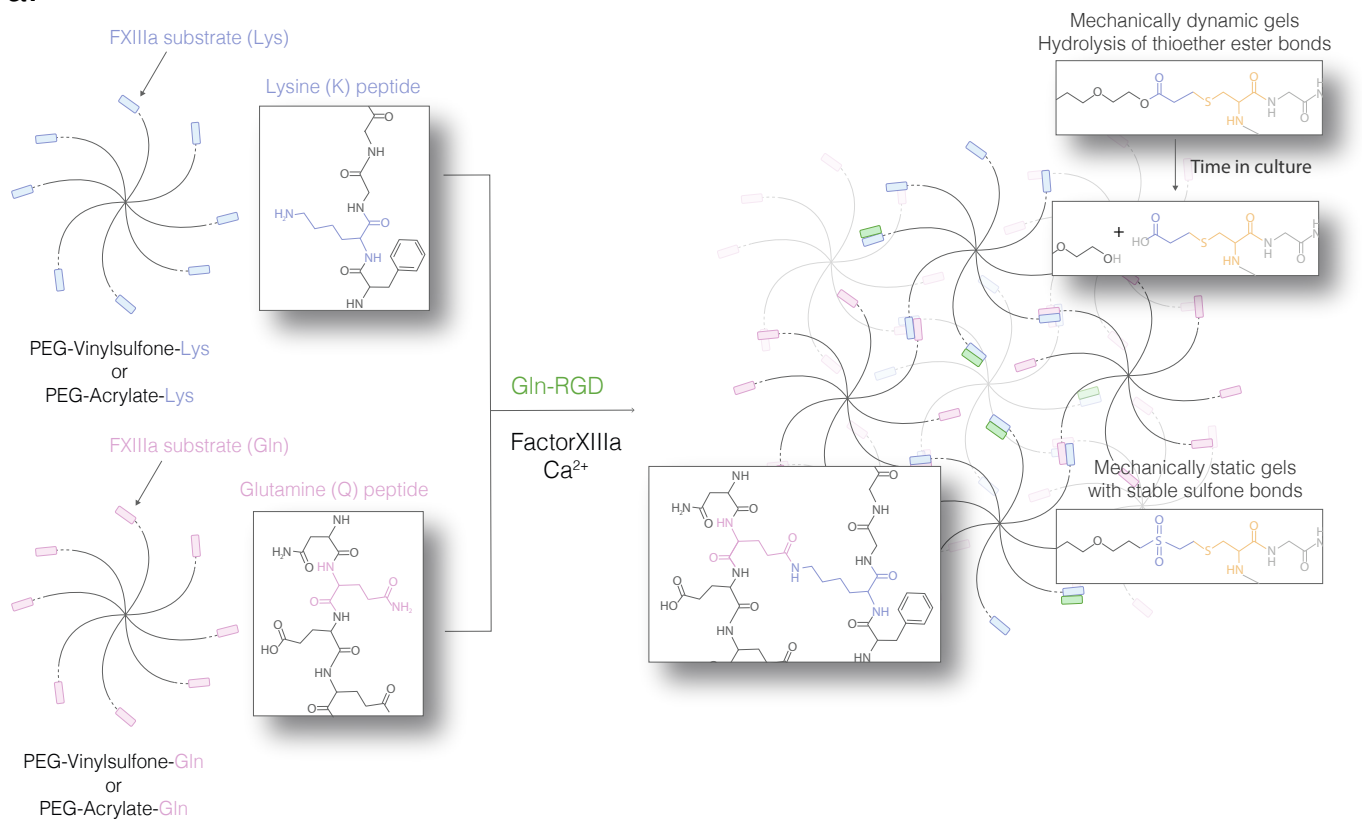

b.

ISC expansion

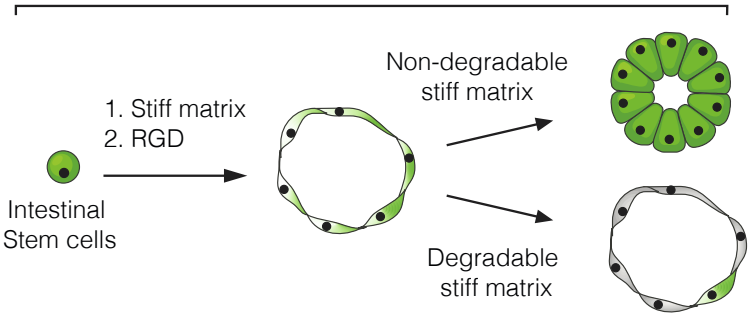

Organoids formation

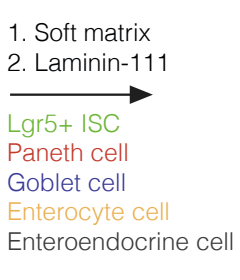

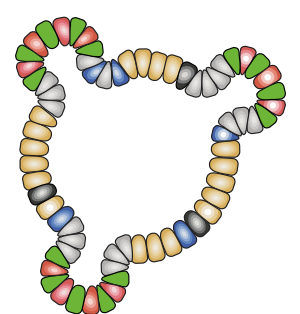

C.
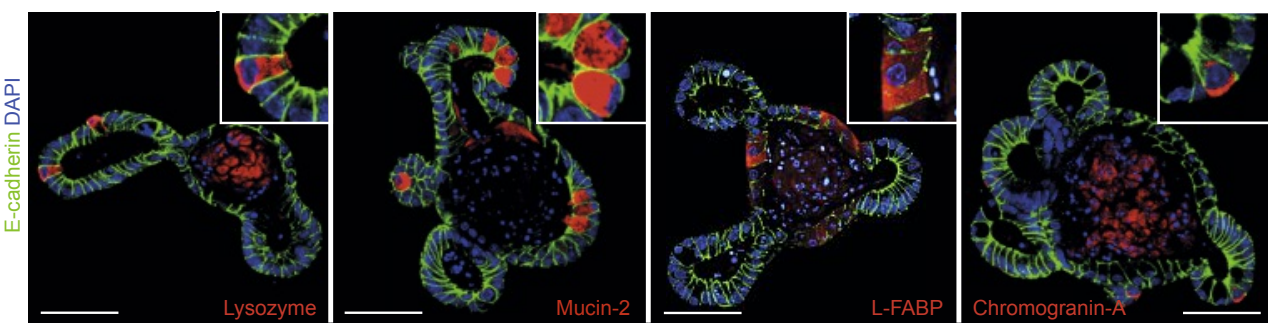
ity, and macromolecular diffusion). Often referred to as 'blank canvas' or 'blank slate', covalently crosslinked gels are typically inert, that is, they do not interact with cells unless they are chemically modified with desired cell recognition sites which confer cell adhesion and/or specific cell-signaling and cell-responsive properties. ${ }^{[15-17]}$ For instance, integrin-binding peptides such as Arg-Gly-Asp (RGD) can be grafted into the networks to render them cell-adhesive, or proteolytically sensitive domains (such as GPQGIWGQ) can be incorporated in such hydrogels, ${ }^{[18]}$ allowing customizable cell-mediated degradation by proteases such as matrix metalloproteases (MMPs). The modularity of such synthetic hydrogels is one of the major advantages that can be exploited to systematically 'deconstruct' the complexity of native ECMs to study the effect of different biochemical and physical cues on cell fate and organoid development. ${ }^{[19-21]}$

Our group reported the first well-defined synthetic hydrogel as Matrigel ${ }^{\mathrm{TM}}$ alternative for mouse and human intestinal organoids. ${ }^{[19,22]}$ (Fig. 1). We used PEG-based gels crosslinked via activated factor XIII, a transglutaminase that is responsible for polymerizing fibrinogen into stable fibrin gels. To promote ISC survival and adhesion via integrin-binding, these gels were functionalized with the fibronectin-derived adhesion peptide RGDSP as well as laminin-1 (Fig. 1a), two ECM cues that were crucial to promote robust ISC expansion (RGDSP alone) and organoid formation (RGDSP and laminin-1), respectively. The gels' mechanical properties (Young's modulus) turned out to play a key role at multiple steps of organoid formation, with an intermediate stiffness being optimal for ISC expansion (under self-renewal culture conditions) and matrix softening being critical for induc- ing crypt formation (under differentiation conditions) from stem cell colonies. Intriguingly, mechanically softening hydrogels, obtained through ester-based hydrolysis of a fraction of the covalently crosslinked PEG network, was required to promote crypt formation. Notably, the ability to precisely modulate the physical and biochemical properties of these synthetic gels allowed us to identify the role of different parameters of cellular microenvironment in promoting intestinal stemness and organoid formation (Fig. 1b,c).

Garcia and colleagues reported a similar concept for the 3D culture of human pluripotent stem cell-derived intestinal organoids $^{[23]}$ (Fig. 2). By using PEG macromers crosslinked via Michael-addition (using end-functionalized PEGs bearing thiol and maleimide residues) (Fig. 2a), these authors identified optimal conditions for intestinal stem cell expansion and differentiation to similar levels compared to Matrigel ${ }^{\mathrm{TM}}$ (Fig. 2b,c). In addition, they showed that these synthetic gels support the development of other human organoids such as those of the lung, and could confirm that epithelial tissue growth, polarization and lumen formation occurs within a relatively narrow range of ECM elasticity.

\section{Building Next-generation Bioinspired Hydrogels for Organoid Culture}

A notable drawback of covalently crosslinked hydrogels for organoid culture is that they do not recapitulate the dynamic physical characteristics of native ECMs: Because these gels in essence behave as linear elastic networks, organoid expansion and morphogenesis may be physically impaired by the build-up of substantial compressive forces that cannot be dissipated. ${ }^{[19]}$ What a.

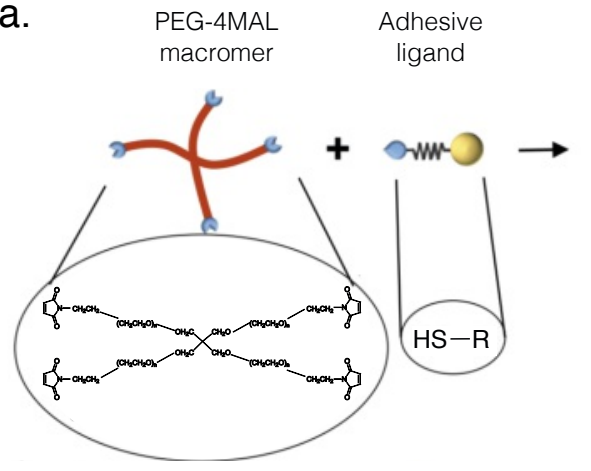

\section{Functionalized PEG-4MAL}

Protease-degradable crosslinking peptide

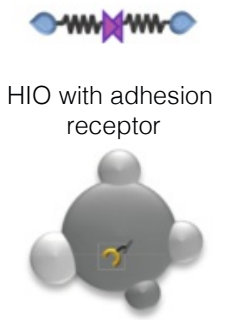

Michael-type addition reaction I
I
I
I

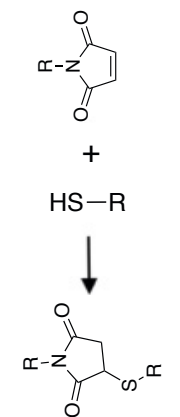

Fig. 2. Synthetic PEG-based hydrogels engineered as niches for human ISC expansion and organoid formation. a) Schematic illustrating the chemical structure of the synthetic hydrogels formed via Michael-type addition. b) Summary of the protocol for the derivation of human intestinal organoids. c) Intestinal organoids formed in Michael-type addition hydrogels with key differentiated cell types. Figures adapted with permission from Macmillan Publishers Ltd. ${ }^{[23]}$ b.
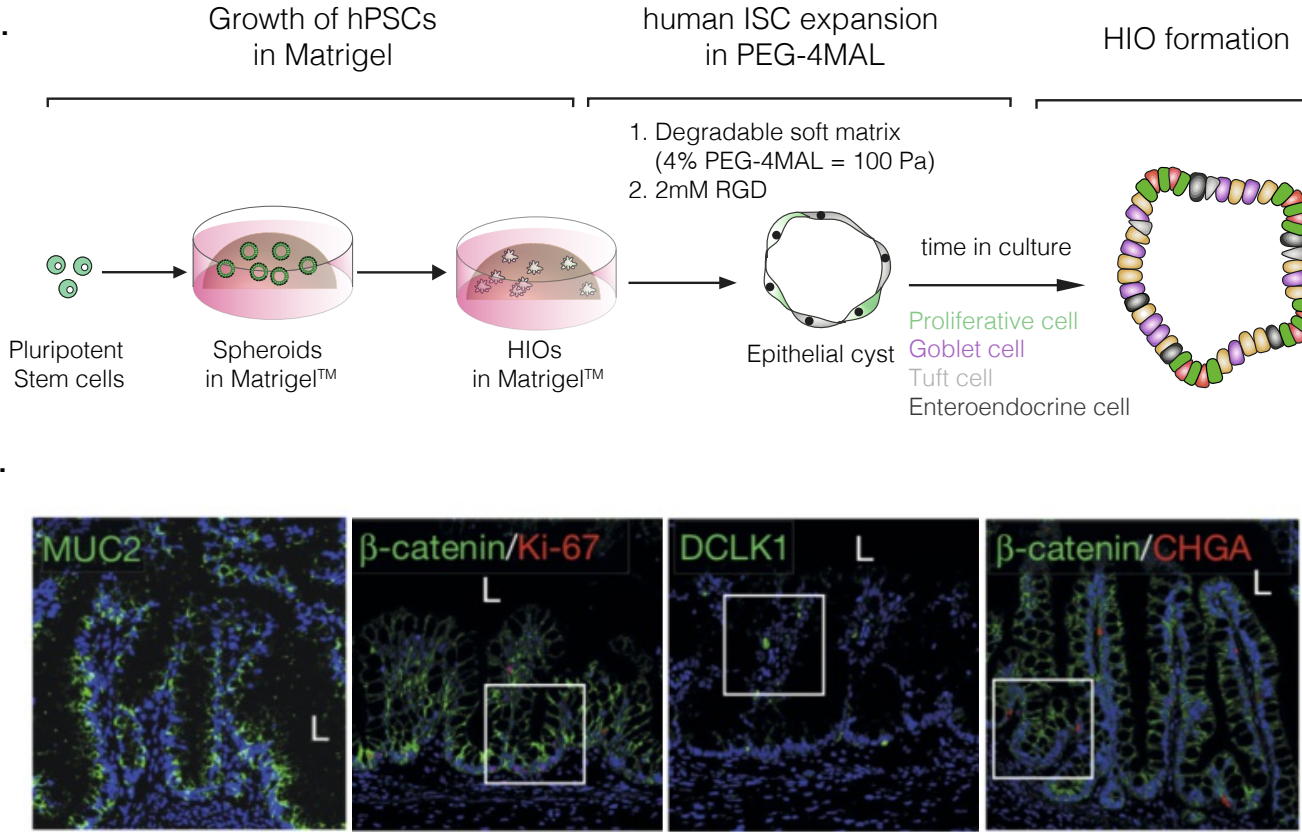

HIO formation in PEG-4MAL

1. Degradable soft matrix

(4\% PEG-4MAL = $100 \mathrm{~Pa})$ 2. $2 \mathrm{mM}$ RGD

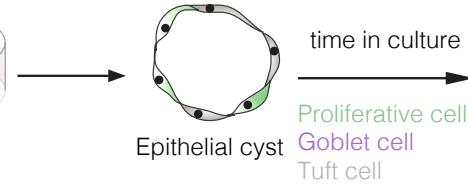

Enteroendocrine cell

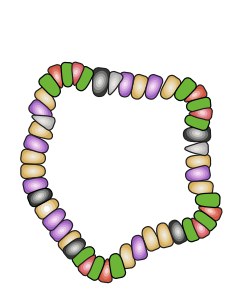


is more, the irreversible nature of the break-down mechanisms of the covalent bonds, either through ester-based hydrolysis, ${ }^{[19]}$ or MMP-mediated proteolytic degradation, ${ }^{[19,23]}$ may prevent longterm organoid culture.

A potentially fruitful strategy to solve these problems is to synthesize hydrogels that are crosslinked, fully or partially, by physical interactions ${ }^{[24,25]}$ (Fig. 3a). These hydrogel types are often referred to as 'reversible' gels that, compared to covalently crosslinked gels, are held together by much weaker transient interactions stemming for example from hydrogen-bonding, [26] ionic ${ }^{[27]}$ or hydrophobic interactions. ${ }^{[28]}$ Reversible hydrogels are often sensitive to variations of extrinsic parameters such as $\mathrm{pH}$, temperature, or ionic strength that can trigger reversible changes in crosslinking and thus matrix mechanics. ${ }^{[29]}$ The reversible nature of the physical crosslinks brings very interesting dynamic characteristics such as shear-thinning or self-healing, physicochemical behaviors that are reminiscent of native ECMs. We expect that physically crosslinked hydrogels will allow improved organoid development, potentially resulting in larger tissue sizes and more elaborate morphogenetic responses.

A second interesting hydrogel engineering strategy for improved organoid culture is the use of photo-modulatable synthetic hydrogels (Fig. 3b). That is to say, most organoids develop through stochastic processes in which initially relatively homogeneous populations of cells break symmetry to give rise to spatially distinct domains containing other cell types. For example, in the intestine, the stochastic appearance of a Paneth cell constitutes the symmetry-breaking event that transforms the circular tissue into a budding, crypt-containing organoid.[30,31] Wnt and Notch signaling have been demonstrated to be critical for initiating and maintaining crypt architecture. In this context, light-mediated patterning (or alternatively, microfluidic technology) could be used to locally display Wnts, Notch ligands or other morphogenetic signals to trigger crypt formation at specific locations through morphogen signaling-induced symmetry-breaking. Furthermore, the dependency of budding on matrix mechanics ${ }^{[19]}$ could be exploited to induce symmetry breaking and crypt formation in a deterministic manner by light-mediated and localized modulation of hydrogel mechanics. Anseth and co-workers (as well as many other groups) have, for example, developed strategies to use light to dynamically modulate hydrogel mechanics and chemistry.[24,32] Indeed, by using multiphoton microscopy, both chemical and physical properties could be tuned independently, potentially allowing to control multiple stages of in vitro organogenesis in real-time and at specific locations, providing exciting perspectives towards an era of 'synthetic morphogenesis'. However, it should be noted that although photo-modulatable synthetic hydrogels represent exciting research tools, their broader application, e.g. in drug discovery or personalized medicine, is likely limited; alternative approaches such as those based on micro-engineering could complement smart synthetic hydrogels in the quest to improve organoid culture and applicability.

\section{Conclusion}

Owing to their intriguing capacity to mimic several physiological hallmarks of native tissues and organs, organoids hold great promise for numerous applications in biology and biomedicine. However, a broader translation of organoid technology from the
Fig. 3. Bioinspired synthetic hydrogels for 3D organoid culture and manipulation. a) Schematics illustrating how matrix mechanics may influence morphogenesis arguing for a need for dynamic (i.e. reversible and self-healing) gels readily accommodating tissue growth. b) Schematic highlighting the use of light-mediated gel patterning to direct cell fate and morphogenesis in time and space. a.

Covalently crosslinked gel
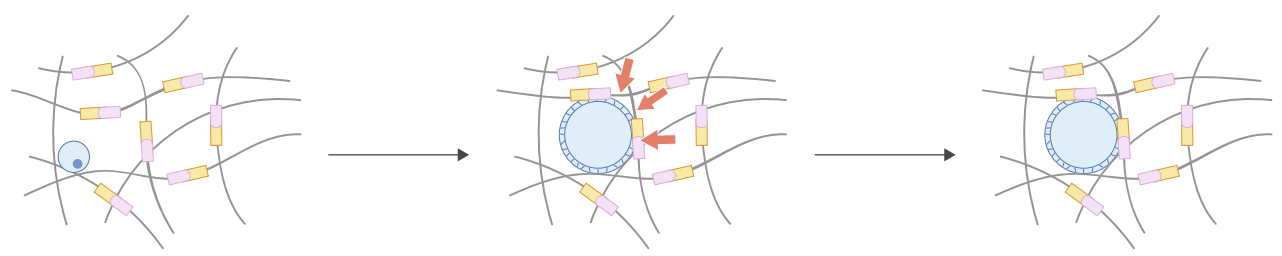

Physically crosslinked gel

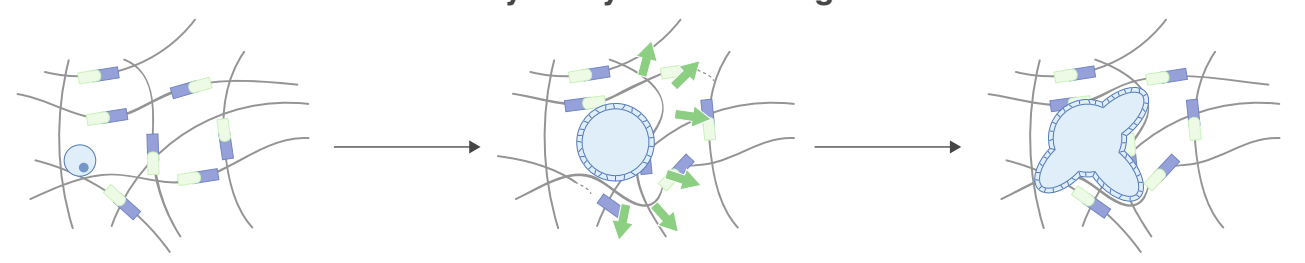

b.

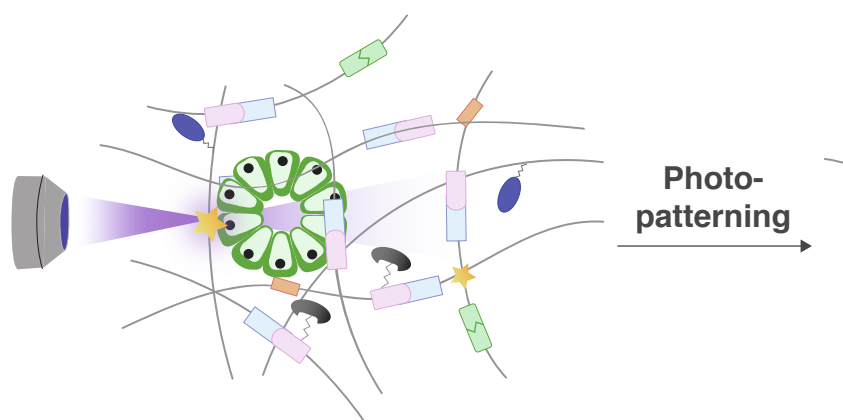

Structural components

Bioactive domains

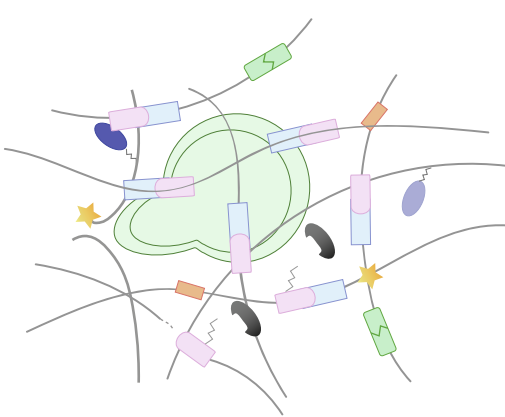

Photo-modulatable domains

Photodegradable unit e.g. Nitrobenzyl ether

Multiarm PEG (e.g. fibronectin, RGD)

Cell signaling cue (e.g. growth factor) 
lab into real-life applications is hampered by the dependency on relatively archaic cell culture technology. The design and application of synthetic, bio-inspired hydrogels for growing stem cells in more defined and modulatable 3D microenvironments could provide a path forward in rationally guiding in vitro morphogenesis.

\section{Acknowledgements}

We thank the National Center of Competence in Research (NCCR) Bio-Inspired Materials for financial support.

Received: November 16, 2018

[1] H. Clevers, Cell 2016, 165, 1586, doi: 10.1016/j.cell.2016.05.082.

[2] M. A. Lancaster, J. A. Knoblich, Science 2014, 345, 1247125, doi: 10.1126/ science. 1247125 .

[3] T. Sato, R. G. Vries, H. J. Snippert, M. van de Wetering, N. Barker, D. E. Stange, J. H. van Es, A. Abo, P. Kujala, P. J. Peters, H. Clevers, Nature 2009, 459, 262, doi: 10.1038/nature07935.

[4] T. Sato, H. Clevers, Science 2013, 304, 1190, doi: 10.1126/science.1234852.

[5] M. Eiraku, N. Takata, H. Ishibashi, M. Kawada, E. Sakakura, S. Okuda, K. Sekiguchi, T. Adachi, Y. Sasai, Nature 2011, 472, 51, doi: 10.1038/nature09941.

[6] M. Eiraku, K. Watanabe, M. Matsuo-Takasaki, M. Kawada, S. Yonemura, M. Matsumura, T. Wataya, A. Nishiyama, K. Muguruma, Y. Sasai, Cell Stem Cell 2008, 3, 519, doi: 10.1016/j.stem.2008.09.002.

[7] G. Rossi, A. Manfrin, M. P. Lutolf, Nat. Rev. Gen. 2018, 19, 671, doi: 10.1038/s41576-018-0051-9.

[8] M. Huch, B. K. Koo, Development 2015, 142, 3113, doi: 10.1242/ dev. 118570 .

[9] H. K. Kleinman, G. R. Martin, Sem. Cancer Bio. 2005, 15, 378, doi: 10.1016/j.semcancer.2005.05.004.

[10] M. Huch, J. A. Knoblich, M. P. Lutolf, A. Martinez-Arias, Development 2017, 144, 938, doi: 10.1242/dev.150201.

[11] N. Gjorevski, A. Ranga, M. P. Lutolf, Development 2014, 141, 1794, doi: 10.1242/dev.101048.

[12] A. Ootani, X. Li, E. Sangiorgi, Q. T. Ho, H. Ueno, S. Toda, H. Sugihara, K. Fujimoto, I. L. Weissman, M. R. Capecchi, C. J. Kuo, Nat. Med. 2009, 15, 701, doi: $10.1038 / \mathrm{nm} .1951$.

[13] N. Sachs, Y. Tsukamoto, P. Kujala, P. J. Peters, H. Clevers, Development 2017, 144, 1107, doi: 10.1242/dev.143933.
[14] N. Broguiere, L. Isenmann, C. Hirt, T. Ringel, S. Placzek, E. Cavalli, F. Ringnalda, L. Villiger, R. Züllig, R. Lehmann, G. Rogler, M. H. Heim, J. Schüler, M. Zenobi-Wong, G. Schwank, Adv. Mater. 2018, 165, 1801621, doi: 10.1002/adma.201801621.

[15] D. Seliktar, Science 2012, 336, 1124, doi: 10.1126/science.1214804.

[16] M. W. Tibbitt, K. S. Anseth, Biotechnol. Bioeng. 2009, 103, 655, doi: 10.1002/bit.22361.

[17] M. P. Lutolf, J. A. Hubbell, Nat. Biotechnol. 2005, 23, 47, doi: 10.1038/ nbt 1055.

[18] M. P. Lutolf, J. L. Laver-Fields, H. G. Schmoekel, A. T. Metters, F. E. Weber, G. B. Fields, J. A. Hubbel, Proc. Natl. Acad. Sci. USA 2003, 100, 5413

[19] N. Gjorevski, N. Sachs, A. Manfrin, S. Giger, M. E. Bragina, P. OrdóñezMorán, H. Clevers, M. P. Lutolf, Nature 2016, 539, 560, doi: 10.1038/nature20168.

[20] A. Ranga, S. Gobaa, Y. Okawa, K. Mosiewicz, A. Negro, M. P. Lutolf, Nat. Commun. 2014, 5, 4324, doi: 10.1038/ncomms5324.

[21] A. Ranga, M. Girgin, A. Meinhardt, D. Eberle, M. Caiazzo, E. M. Tanaka, M. P. Lutolf, Proc. Natl. Acad. Sci. USA 2016, 113, E6831, doi: 10.1073/ pnas. 1603529113

[22] N. Gjorevski, M. P. Lutolf, Nat. Protoc. 2017, 12, 2263, doi: 10.1038/ nprot.2017.095.

[23] R. Cruz-Acuña, M. Quirós, A. E. Farkas, P. H. Dedhia, S. Huang, D. Siuda, V. García-Hernández, A. J. Miller, J. R. Spence, A. Nusrat, A. J. García, Nat. Cell Biol. 2017, 19, 1326, doi: 10.1038/ncb3632.

[24] A. M. Rosales, K. S. Anseth, Nat. Rev. Mater. 2016, 1, 10776, doi: 10.1038/ natrevmats.2015.12.

[25] H. Wang, S. C. Heilshorn, Adv. Mater. 2015, 27, 3717, doi: 10.1002/ adma.201501558.

[26] P. Y. W. Dankers, M. C. Harmsen, L. A. Brouwer, M. J. A. Van Luyn, E. W. Meijer, Nat. Mater. 2005, 4, 568, doi: 10.1038/nmat1418.

[27] X. Zhao, N. Huebsch, D. J. Mooney, Z. Suo, J. Appl. Phys. 2010, 107, 063509, doi: 10.1063/1.3343265.

[28] M. Mihajlovic, M. Staropoli, M.-S. Appavou, H. M. Wyss, W. PyckhoutHintzen, R. P. Sijbesma, Macromol. 2017, 50, 3333, doi: 10.1021/acs. macromol.7b00319.

[29] X. Li, X. Su, J. Mater. Chem. B 2018, 6, 4714, doi: 10.1039/C8TB01078A.

[30] T. Sato, H. Clevers, Science 2013, 340, 1190, doi: 10.1126/science.1234852.

[31] H. F. Farin, I. Jordens, M. H. Mosa, O. Basak, J. Korving, D. V. F. Tauriello, K. de Punder, S. Angers, P. J. Peters, M. M. Maurice, H. Clevers, Nature 2016, 530, 340, doi: 10.1038/nature16937.

[32] A. M. Kloxin, A. M. Kasko, C. N. Salinas, K. S. Anseth, Science 2009, 324, 59, doi: 10.1126/science.1169494 